\title{
EAl Endorsed Transactions

\section{Level-6 Automated IoT integrated with Artificial Intelligence Based Big Data-Driven Dynamic Vehicular Traffic Control System}

\author{
Maria Michael Visuwasam L ${ }^{1, *}$, Ashwin Balakrishna ${ }^{1}$, Nikitha Keerthana S R ${ }^{1}$ and Kowsalyaa $\mathrm{V}^{1}$ \\ ${ }^{1}$ Computer Science and Engineering, Rajalakshmi Institute of Technology, Chennai, Tamilnadu, India.
}

\begin{abstract}
The current traffic control system (TCS) is not the most efficient system present for regulating traffic. Hoping to solve this we come up with dynamic data recording systems which encompasses of RFID tag and reader. The traffic density at each lane is calculated based on count of RFID's apprehended. Depending on the density, the TCS is assigned a value of 15 to 70 seconds in round robin method for control of vehicular congestion. This proposed model also uses image processing for the detection of ambulances and also an active RFID for tracking the real-time location of these assets or in high-speed environments such as that of tolling. This allows the passage of ambulances through dense traffic. This system hopes to achieve to reduce the needless wait of crowded side, to reduce the long traffic chains, and to allow emergencies (medical/ vigilante) quickly through the traffic. The major merits of the system are it prevents unnecessary waiting time when no cars are present at the opposite route, gives the commuting passengers a better and more comfortable driving experience through their journey.
\end{abstract}

Keywords: RFID, Energy Efficient Device-to-Device (D2D) Communications, active, Energy Efficient Routing Protocols, round robin. Received on 07 April 2020, accepted on 24 April 2020, published on 29 April 2020

Copyright (C) 2020 Maria Michael Visuwasam L et al., licensed to EAI. This is an open access article distributed under the terms of the Creative Commons Attribution licence (http://creativecommons.org/licenses/by/3.0/), which permits unlimited use, distribution and reproduction in any medium so long as the original work is properly cited.

doi: 10.4108/eai.13-7-2018.164176

*Corresponding author. Email: micael vm@yahoo.co.in

\section{Introduction}

Driving in India has been governed by various legal powers and in a few cases, it is a subject to the passing of a driving test. The Ministry of Road Transport and Highways a branch of the Government of India, is the apex body for formulation and administration of these rules, regulations and laws relating to road transport, national highways and transport research, in order to increase the mobility and efficiency of the road transport system in India we have come up with the following Traffic Control System . The existing traffic control system works using a round robin rule of 30 seconds which causes a lot of vehicular congestion.
This leads to the problem of traffic in lanes causing the obstruction of ambulances, and fire engines. This leads to loss of life and other major casualties. The traffic also produces many kinds of pollution. [1]

This proposal overcomes these disadvantages using RFID tags and IR sensors. The RFID tags are read from the vehicles and the signal to go is assigned in a round robin method (clockwise) from 15 seconds to 70 seconds. This also reduces the time allocation for lanes without any vehicles and reduces the usage of electricity.

The lanes are assigned by the count of vehicles in that lane by scanning the passive RFID tags in transportation vehicles. If the lane has no vehicular 
passive RFID tag being read then, the signal allocation is backtracked and assigned to the most traffic congested lane. [3]

The ambulances, fire engines and patrol vehicles have active RFID tags that allow the recognition of these vehicles uniquely. These vehicles are read and the signal is changed to $\mathrm{GO}$ which allows the free passage of the active vehicles. This feature also includes image processing to identity the vehicles. The patrol vehicles are also included occasionally in vigilante security. [13] The time taken by these vehicles to pass the signals is most important as they are dispatched to save lives. This backtracking round robin method changes the signal to GO immediately when the active RFID tag is read in any lane irrespective of which lane GO signal has at present. Machine learning is used in the process of understanding the time required for the vehicles to pass the particular signal at different variables of time and traffic in the area. This is added to the training data in real time and the recordings are saved. [10]

The proposed model also allows the traffic police to supervise from a remote location rather than being at every signal and facing immense problems in controlling the traffic. This unsupervised method reduced the human workload and increases productivity immensely. [2]

\section{Working of The System}

\subsection{Reading of the vehicles}

The vehicle in each lane has its own RFID tag installed which can be read using a RFID reader. These readings can detect two wheelers, four wheelers, heavy duty vehicles, etc. This is done to find the density of the vehicular congestion in any particular signal. [4]. An RFID reader is placed very placed very strategically at the the sides of the railing of the lanes. This is done so that we can achieve a maximum distance or range to check the presence of maximum number of cars. A Jaguar Platinum RFID reader gives us a range of about $90 \mathrm{ft}$ which sums up to a distance of approximately 27 meters and setup with 2 of these can cover upto 60 meters which can easaily count upto $30-40$ cars in a lane. Even if there are heavy load vehicles present in the system the count of the number of RFID remains the same and the calculations would still remain perfectly fine.

\subsection{Sensors used in the process of detection}

The two sensors used in the detection of the vehicles are:

- Radio Frequency Identification (RFID) tag

- $\quad$ Infra-red (IR) sensor.

\subsection{Implementation of the Sensors}

The RFID tag installed in each vehicle is read within a particular range using RFID reader. This can detect two wheelers, four wheelers, heavy duty vehicles, etc. [6] the aforementioned vehicles have passive RFID tags that do not actively track the live location in high-speed environments. This is opted because of the calculation of the density of traffic alone. The active RFID tags used in the ambulances, fire engines, police patrol vehicles, etc. are read for the live tracking in the most traffic congested areas. These sensors are static and do not provide data for the machine learning techniques. [5]

The IR sensors are used to take the count of vehicles passing the signal. The sensor senses in a single line and the count is recorded in real-time. This is the most effective sensor used for collecting the data to be trained. This sensor is dynamic and the data can be used in machine learning techniques.

\subsection{Machine Learning}

The data from the IR sensors are recorded in real-time. This data holds the records of the time taken by the vehicles in a particular lane to cross the signal with the density of the traffic. The theoretical observation which states a definite number of seconds for the vehicles to pass by a signal can be verified and updated using the principle of variable change for calculation of duration. The Machine Learning Algorithm used here is that of SVM(Support Vector Machine). Being an algorithm which helps in efficient classification of two group data, we believe it is the best suited method for acquiring this data from the system. [7]. The use of cloud database is essential for the proposed system as it requires maximum amount of space for the acquisition of realtime data which is of about 10 data/second .Hence we prefer the use of a NoSQL cloud database such as that of Rack-space, since it incorporates a SAN storage network based on the OpenStack platform. These are used only for the first few months as we require It only during the training period and after that we would use a local database as it reduces the cost of the system. There is no actual dataset present for the calculation of the following parameters and hence we use a dynamic database which we in turn train with respect to the input data using the Machine Learning algorithms such as that of Support Vector Machine(SVM), which a a well known for its high success ratio and better predictability using the clustering and classification techniques.

\subsection{Efficiency}

The efficiency of the system is based on major factors such as:

- The number of vehicles using RFID tags, older vehicles doesn't have RFID tags (vehicles manufactured before 2012). 
- The range of RFID sensor, the sensor should implement in such a way that it covers only the particular (left/right) side of the lane.

The efficiency of the system will be maximum when the number of vehicles on side of the lane is more in number than other lanes. [8]

\section{Working of The Sensor}

The proposed system contains the following sensors: RFID reader, IR sensor and RFID tags which are mounted on the vehicles. The RFID reader uses electromagnetic fields to identify and automatically track tags attached to the vehicles. When a vehicle with RFID tag passes over the range of area covered by the RFID reader, it gets triggered by the nearby RFID reader's electromagnetic pulse. The RFID tag now transmits the data to the RFID reader which consists of the vehicle identity number. The passive RFID tags are used in normal vehicles; these are powered by the electromagnetic signals from the RFID reader's electromagnetic pulse. Active RFID tags are used in emergency vehicles such as an ambulance. These active RFID tags have their own batteries supplying power which enables them to be identified when approaching in a greater distance. [9]

When a vehicle gets in to the range of an RFID reader (usually 600 meters from the reader) the RFID tags gets triggered and sends data to the reader. The RFID reader receives such data from all the vehicles in its range. Two RFID readers are used in a side of the road to get an approximate value of the number of vehicles. The IR sensors are used to check whether all the vehicles have passed through the junction. The IR sensors detect the obstacles by sending a continuous beam of infrared signal which is detected by the infrared detector on the opposite side. Thus, when there is no vehicle passing through the road for a particular amount of time, being level-6 automation, the signal gets switched to the next side. This switching happens in a round robin manner. In traditional manner this round robin slots are 30 seconds for each lane. So once when the vehicles in a particular lane have crossed the junction with the data from the IR sensor, the RFID reader generates a trigger to the signal which switches the signal to the desired side of the lane. [14]

Another feature is that when an ambulance is approaching in any side of the road then the RFID readers detect these signals specifically as they use active RFID tags and switches the signal to green to the corresponding side.

The following system proposes a dynamic system of data collection manipilation and extraction and hence does not comprise of an offset value The density of a particulr lane is calculated using a simple formulae of
The principle of variable change is only applicable for static data and not for dynamic ones. Hence it is not considered to have affected or brought about a change in the results of the prediction of the proposed model in the following scenerio.

\subsection{Equations}

\section{PSEUDOCODE:}

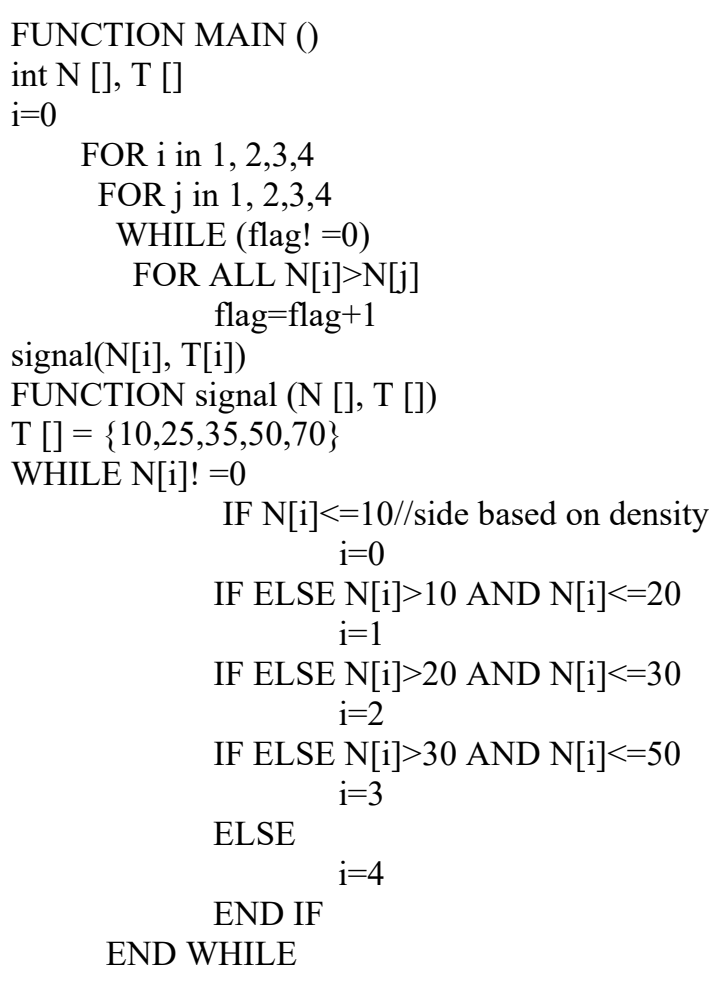

OUTPUT "SIGNAL ON LANE N[i] IS GREEN FOR $\mathrm{T}[\mathrm{i}]$ secs" 


\subsection{Models and Explanation}

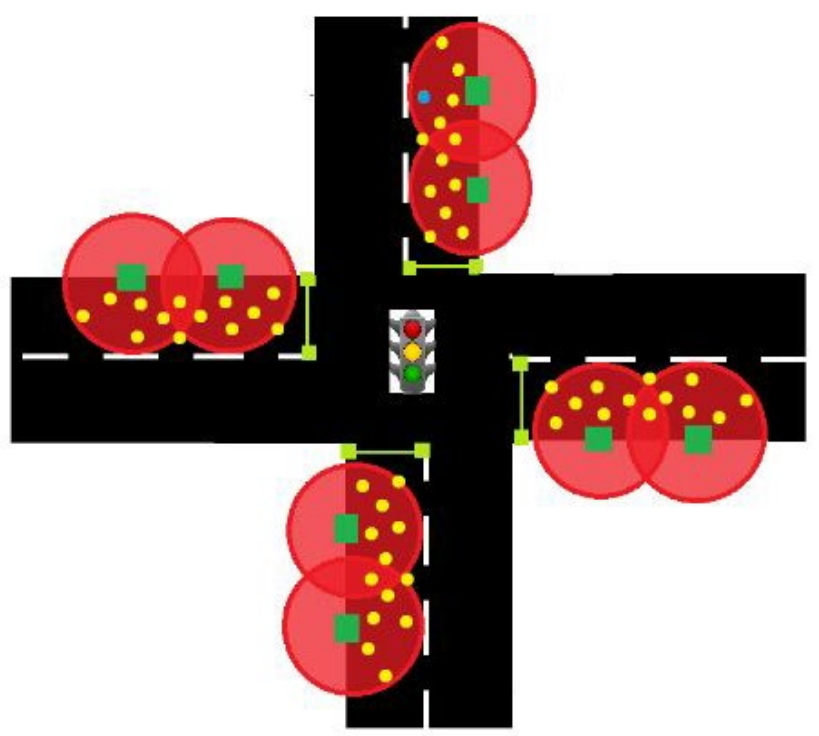

Figure 1. Representation of the System

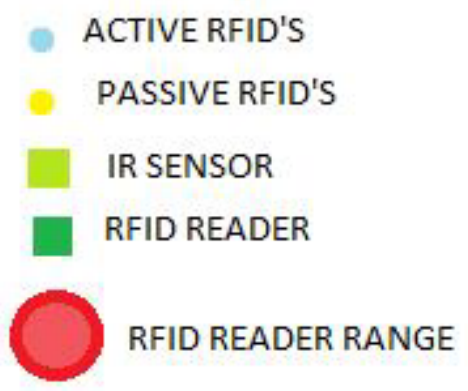

Figure 2. Legend

The following system uses a density based principle of variable change mechanism to control the flow of traffic at signal junctions. Following the traditional method of round robin rule every traffic system uses a 30 seconds per side style system for controlling traffic. But how efficient is this when it comes to that side of the road which has very few or absolutely no cars present in that lane. [13]

As shown in Fig.1 the system uses the RFID present on the vehicles to detect and calculate the presence of the number of vehicles in the present lane and based on their density they decide whether or not to cut down on time based on previous calculations performed by the system which having been previously tuned or trained using machine learning techniques such as SVM(Support Vector Machine) and reinforcement learning to make the system understand better on how long has to be given to the side of the signal based on the density of each side of the signal. The IR sensor present at the start of the road helps us identify whether or not all cars have passed from that side of the road as it helps us read data dynamically which is not possible in the case of an RFID since it gives us a static data. Based on all these aspects and previous results the system would a lot the best possible time limit required to pass through. [12]

Another feature in the proposed system is that of the active and passive RFID that will be used by the system to identify whether or not an ambulance or a vigilantly vehicle has made its presence in the range. Being a level 6 automated system, it finds out on its own and switches it's activity from the green signal on another road to the road where the ambulance or vigilantly vehicle is and provides all necessary assistance it requires. [11]

Equipment Used:

- $\quad$ M3 Texas instruments ARM Cortex M4,

- TMS320C28X core ARM microcontroller,

- E18-d80nkIR sensor - Potential Labs,

- UHF (Ultra High Frequency)RFID reader.

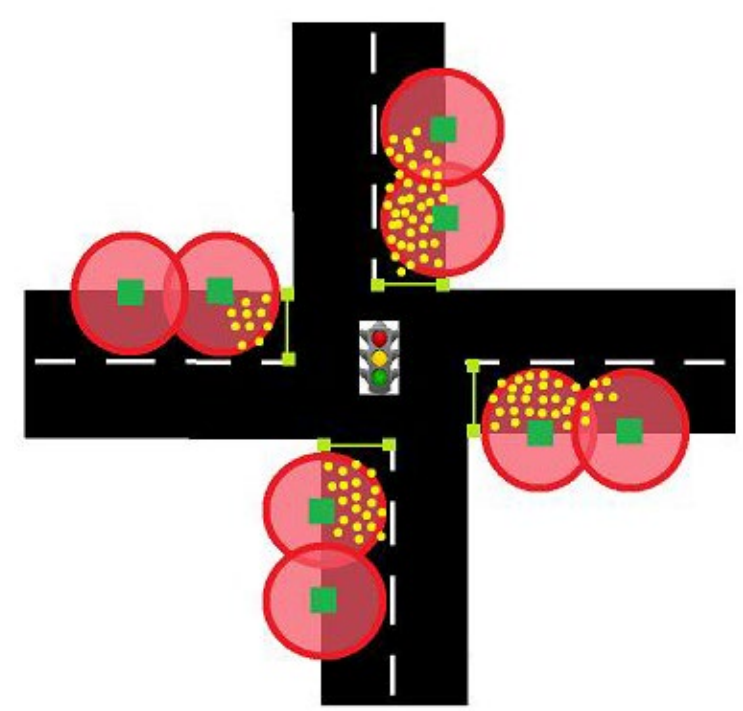

Figure 3. Iteration 1: Initial State

Consider this as shown in Fig. 2 as the initial state. The lane in the north consists of 40 vehicles (say), now when the signal is switched to green; the vehicles pass by at the rate of 1 vehicle/second for 30 seconds. Thus, at the end of 30 seconds, 30 vehicles would have crossed. This is represented in the Fig. 3. [10] 


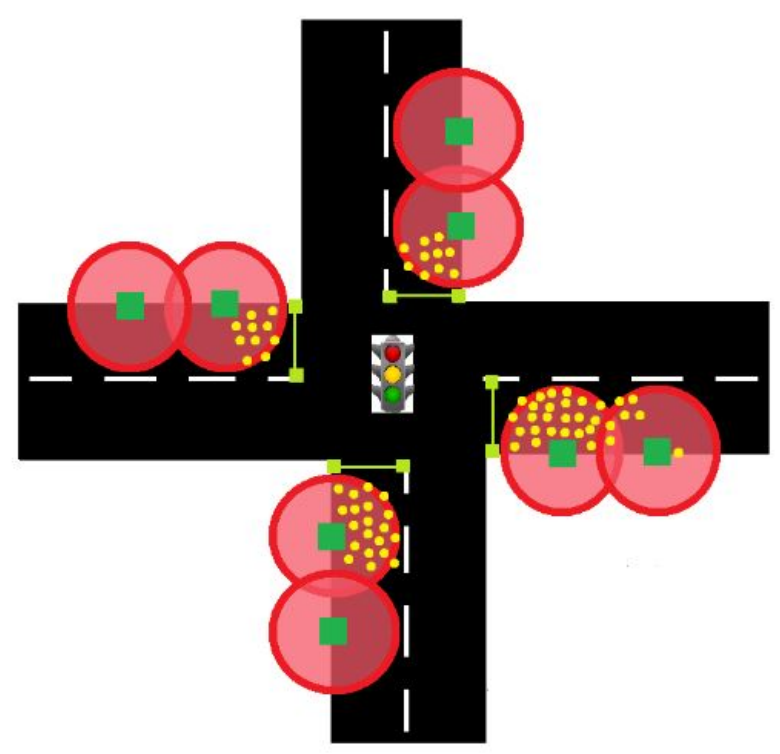

Figure 4. Iteration 2:post 30 seconds -signal on the north lane has been opened

Now, in round robin fashion the signal is switched to green for the east lane, say there are 30 vehicles in this lane. Considering the same conditions as above, after 30 seconds all the vehicles in the lane would have crossed the signal which in Fig. 4.

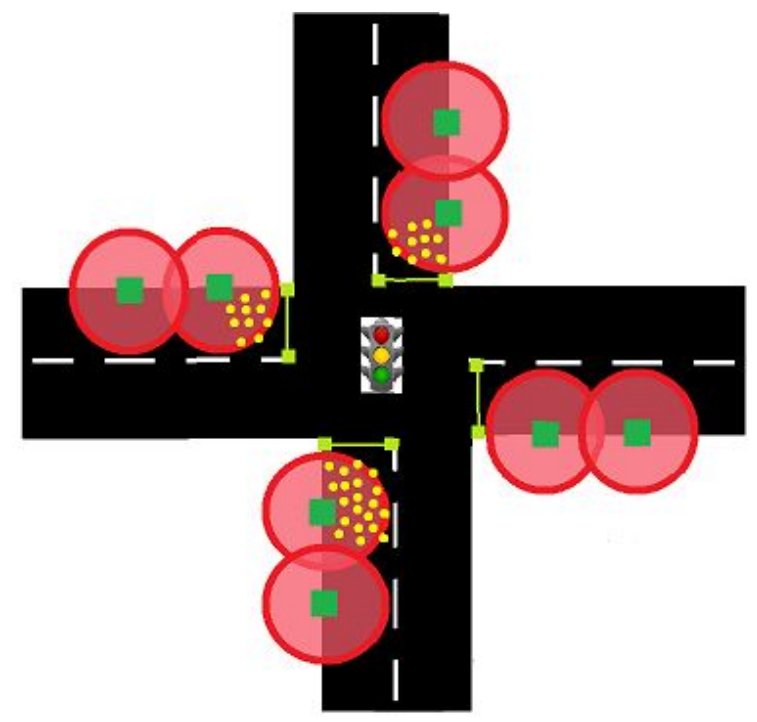

Figure 5. Iteration 3:post 30 seconds -signal on the east lane has been opened

Now, again following the round robin fashion, the signal in the south lane is switched to green for 30 seconds. Say there are 20 vehicles in this lane, the RFID reader collects the data and calculates that the vehicle density is less. Adding on to the data from the IR sensor, that no vehicles have passed, the signal gets switched to the west lane. Thus the time taken for all the vehicles to pass this lane is lesser that the usual time given on each side of the lane. This is represented in Fig. 5 below.

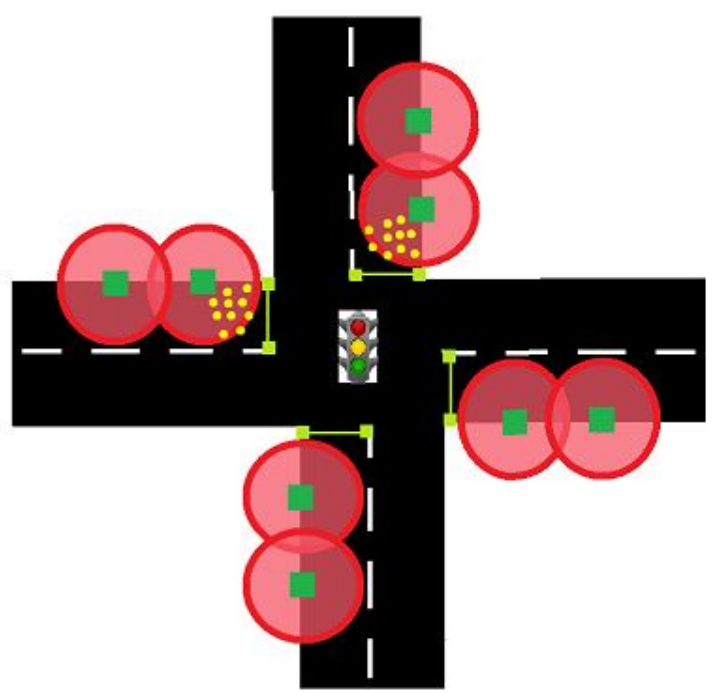

Figure 6. Iteration 4: post 20 seconds -signal on the south lane has been opened

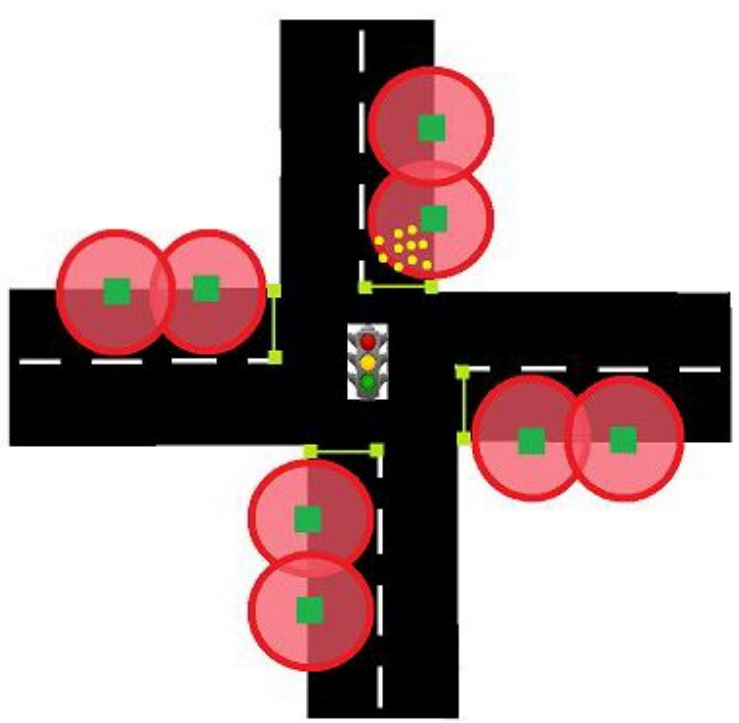

Figure 7. Iteration 5: post 10 seconds -signal on the west lane has been opened

Say that there are 10 vehicles in the west lane (Fig. 6 ) and the vehicles cross the junction within 10 seconds (as per our assumptions), the process similar to the previous lane occurs. The data is collected from the RFID tags and IR sensor, then the signal gets switched to the lane in the north. This occurs repeatedly.

\subsection{Usability of the product}

This system can be implemented in the street junctions of smart cities, highway tollbooths or highway turnings, and in assembly lines of factories. 
Approximate Cost of a System

\begin{tabular}{|c|c|c|c|}
\hline PRODUCT & $\begin{array}{c}\text { Price/Piece in } \\
\text { Rs. }\end{array}$ & $\begin{array}{c}\text { Quanti } \\
\text { ty }\end{array}$ & Rate \\
\hline $\begin{array}{c}\text { ArmCortex- } \\
\text { M } \\
\text { microcontrol } \\
\text { ler }\end{array}$ & 3700 & 1 & 3700 \\
\hline $\begin{array}{c}\text { RFID } \\
\text { readers }\end{array}$ & $\begin{array}{c}25000 \text { (dependi } \\
\text { ng on range) }\end{array}$ & 8 & $\begin{array}{c}2600 * 8=20,8 \\
00\end{array}$ \\
\hline $\begin{array}{c}\text { Cloud } \\
\text { database }\end{array}$ & Free & 1 & 0 \\
\hline Total & & & 24,500 \\
\hline
\end{tabular}

Acknowledgement. Authors would like to thank Mr. Maria Michael Visuwasam for his consultations in domain knowledge and providing us with real road traffic data. Authors would also like to acknowledge the in-kind support from Rajalakshmi Institute of Technology.

\section{References}

[1] I. Lana, J. D. Ser, M. Velez, and E. I. Vlahogianni, "Road traffic forecasting: Recent advances and new challenges,” IEEE Intell. Transp. Syst. Mag.2018

[2] E. I. Vlahogianni, M. G. Karlaftis, and J. C. Golias, "Short-term traffic forecasting: Where we are and where we're going," Transp. Res. C, Emerg. Technol., vol. 43, pp. 3-19, Jun. 2014.

[3] K. N. Qureshi and A. H. Abdullah, "A survey on intelligent transportation systems," Middle-East J. Sci. Res., vol. 15 , no. 5, pp. 629-642, 2013.

[4] C. Khatri, "Real-time road traffic information detection through social media," Jan. 2018

[5] D. Wang, A. Al-Rubaie, J. Davies, and S. S. Clarke, "Real time road traffic monitoring alert based on incremental learning from tweets," in Proc. IEEE Symp. Evolving Auton. Learn. Syst. (EALS), Dec. 2014, pp. 50-57.

[6] P.-T. Chen, F. Chen, and Z. Qian, "Road traffic congestion monitoringin social media with hinge-loss Markov random fields," in Proc. IEEE Int. Conf. Data Mining, Dec. 2014, pp. 80-89.

[7] M. J. Lighthill and G. B. Whitham, "On kinematic waves. II. A theory of traffic flow on long crowded roads," Proc. Roy. Soc. London, Ser. A, Math. Phys. Sci., vol. 229, pp. 317-345, May 1955.

[8] C. F. Daganzo, "The cell transmission model. Part I: A simple dynamic representation of highway traffic," Transp. Res. Part B Methodol., vol. 28, no. 4, pp. 269-287, 1994.

[9] Y. Lv, Y. Duan, W. Kang, Z. Li, and F.-Y. Wang, "Traffic flow prediction with big data: A deep learning approach," IEEE Trans. Intell. Transp. Syst., vol. 16, no. 2, pp. 865-873, Apr. 2015.

[10] R. Yu, Y. Li, C. Shahabi, U. Demiryurek, and Y. Liu, "Deep learning:A generic approach for extreme condition traffic forecasting," in Proc. SIAM Int. Conf. Data Mining, Jun. 2017, pp. 777-785.

[11] W. Huang, G. Song, H. Hong, and K. Xie, "Deep architecture for traffic flow prediction: Deep belief networks with multitask learning," IEEE Trans Intell. Transp. Syst.Oct. 2014.

[12] L. Baskar, B. De Schutter, J. Hellendoorn, and Z. Papp, "Traffic control and intelligent vehicle highway systems: A survey," IET Intell. Transp. Syst., vol. 5, no. 1, pp. 38-52, Mar. 2011

[13] M. Haklay and P. Weber, "OpenStreetMap: Usergenerated street maps," IEEE Pervasive Comput., vol. 7, no. 4, pp. 12-18, Oct. 2008.

[14] A. Wegener, M. Piórkowski, M. Raya, H. Hellbrück, S. Fischer, and J.-P. Hubaux, "TraCI: An interface for coupling road traffic and network simulators," in Proc. 11th Commun. Netw. Simulation Symp., Apr. 2008, pp. 155-163.

[15] U. Mori, A. Mendiburu, M. Álvarez, and J. A. Lozano, "A review of travel time estimation and forecasting for advanced traveller information systems," Transportmetrica A, Transp. Sci., vol. 11, no. 2, pp. 119-157, 2015. 